\title{
La homologación de la competencia profesional en el ámbito de los mecanismos alternativos de solución de controversias
}

\author{
The homologation of professional competence in the field of \\ alternative dispute settlement mechanisms
}

Recibido: 20-04-2021 | Aceptado: 08-07-2021

Raquel Rodríguez González*

*Universidad Autónoma de Nuevo León, México

\section{Resumen}

La competencia profesional es actualmente uno de los temas centrales en el contexto de cualquier actividad laboral, ya que dentro de los cambios constantes del contexto social surgen necesidades que exigen calidad de la formación especializada, condiciones propicias que evidentemente favorecen al ámbito de los Mecanismos Alternativos de Solución de Controversias, refiriendo en específico a la intervención del facilitador, la cual es una figura reconocida incluso en la legislación. Esta se ocupa en conducir a los intervinientes en conflicto a la creación de soluciones integrando para la actividad señalada los conocimientos teóricos y prácticos que la ley establece, a la vez de adherir una propia experiencia, competencia y disciplina. El presente artículo reflexiona sobre la necesidad de no perder el enfoque de la homologación de la competencia profesional de quienes en su deber ser y hacer contribuyan a la solución de controversias desde una perspectiva de reconocimiento profesional. Asimismo, se realizan propuestas en relación con la competencia disciplinar, dadas las profesiones diversas que se involucran y que asumen el rol de facilitador lo cual, si no se atiende bajo capacidades delineadas, generarán discrepancias con resultados no deseados, por lo que se resalta en el mismo sobre la importancia de intervenir en dichos procedimientos desde la formalidad profesional, y en un plano de conocimiento homologado y certificado en lo particular.

Palabras clave: Competencia Profesional, Disciplina, Homologado(A), Facilitador, Mecanismos Alternativos De Solución De Controversias.

Cómo citar

Rodríguez González, R. La homologación de la com-

petencia profesional en el ámbito de los mecanismos

alternativos de solucion de controversias. MSC Métodos

De Solución De Conflictos. Recuperado a partir de https://

revistamsc.uanl.mx/index.php/m/article/view/8 


\begin{abstract}
Professional competence is currently one of the central issues in the context of any work activity, since within the constant changes of the social context, needs arise that demand quality of specialized training, favorable conditions that obviously favor the field of Alternative Mechanisms of Dispute Resolution, referring specifically to the facilitator's intervention, which is a recognized figure even in the legislation. This is concerned with leading the parties in conflict to the creation of solutions integrating for the activity indicated the theoretical and practical knowledge that the law establishes, at the same time as adhering to their own experience, competence, and discipline. This article reflects on the need not to lose focus on the homologation of the professional competence of those who in their duty to be and do contribute to the resolution of disputes from a perspective of professional recognition. Likewise, proposals are made in relation to disciplinary competence, given the diverse professions that are involved and that assume the role of facilitator, which if not addressed under outlined capacities, will generate discrepancies with unwanted results, which is highlighted in the same on the importance of intervening in these procedures from the professional formality, and in a plane of homologated and certified knowledge.
\end{abstract}

Key Words: Professional Competence, Discipline, Approved, Facilitator, Alternative Dispute Resolution Mechanisms.

\section{INTRODUCCIÓN}

La competencia profesional homologada al encontrarse vinculada al orden normativo del ámbito de los Mecanismos Alternativos de Solución de Controversias debe en consecuencia asumir el rol disciplinar que se plantea de acuerdo con el orden del procedimiento que lo circunda. Por lo tanto, es importante destacar la descripción visible del desempeño de cada profesional, ya que el requisito para acceder al círculo profesional de dichos mecanismos pudiera resultar un limitante justo por la generalidad del concepto del profesional que se acredita para ocupar tales responsabilidades, mismas que derivan desde la aceptación a dicho compromiso, el cual debe recibirse altamente calificado con la formalidad que merece al encontrarse desde el primer momento de su cometido, de tal forma que se desenvuelva hacia el cauce de la solución del conflicto. Bajo este marco de acción, la sociedad en su expectativa los requiere con calidad, pertinencia y suficiencia en su perfil profesional, así como de conocimientos suficientes que afronten las problemáticas entre particulares inmersos en una sociedad que impulsa desde sus legislaciones medios que ofrezcan certeza jurídica, que contribuyan a la armonía social y que la judicialización de los conflictos entre partes conlleve a crear oportunidades de fortalecer la cultura en la vía de la solución de controversias.

Por tal razón, es que el presente documento tiene el objetivo de analizar la pertinencia de la debida homologación o reconocimien- 
to sobre la competencia profesional que circunda a los mecanismos alternativos y para poder cumplirse se parte de una clarificación de los conceptos y deberes de acuerdo con preceptos teóricos y normativos que dilucidan la necesidad de tener la acción descriptiva de la disciplina específica del facilitador. Ello, desde un enfoque profesionalizante. Esto es, en relación con el ámbito de los mecanismos alternativos y de una competencia profesional homologada. Por tal razón, se debe considerar para estos efectos que como limitante se observa la norma escrita y delineada de forma general que deja de lado la supervisión de resultados y el medio en particular para que el desempeño del facilitador cumpla los requisitos específicos, lo cual es necesario y de gran acierto, dejando a la disposición de la persona física, el medio del desempeño.

A partir de lo anterior, es necesario resaltar tres puntos: a) señalar el marco constitucional que fundamenta su actuar desde un orden normativo local, ya que este apartado es de suma importancia para el profesional que en su formación profesional no tuvo acceso al estudio del marco del Derecho, involucrándolo así desde el primer momento con el fundamento jurídico que tenga como base una homologación de conocimiento; b) destacar el papel de la disciplina de carácter profesional, y su homologación en el ámbito de los mecanismos alternativos, para de esta manera, tener un marco donde señale las acepciones entre la disciplina y lo profesional, ya que la peculiaridad de los requisitos para acceder a una actividad distinta a la formación principal puede ser confusa si no se determina el posicionamiento de tales conceptos; c) enfatizar el elemento de la competencia profesional, que a manera descriptiva expone los requisitos para acceder a formar parte de los procedimientos que implican la certificación sobre el quehacer en el ámbito de la solución de conflictos, mismos que avalan la capacidad en la intervención dentro del cometido en los mecanismos alternativos, destacando a partir de apartados conceptuales que han sido aportados por los teóricos en la materia, los cuales se contrastan con la formalidad de los ordenamientos jurídicos, para que en ambos contextos se proporcione la apertura al fundamento de un enfoque importante sobre la homologación de la competencia.

Es de subrayar que, en los tres puntos precedentes se destaca la importancia de no sólo la expedición de un certificado que avale competencias, también de la actualización constante, donde los interesados sean atendidos para su formación disciplinaria e interdisciplinaria en particular, a la vez de circunscribirse a modelos de actuación donde el juicio de valor personal no impere ante conflictos de terceros, y bajo esta situación se pueda llegar a una conclusión de intervención estratégica, pudiendo aportar planteamientos sobre el contenido, con el propósito de distinguir la importancia de la homologación de una competencia profesional en el ámbito de la solución de problemas, y que con ello se encuentre en relación directa al objetivo jurídico del propósito de los Mecanismos Alternativos de Solución de Controversias. 


\section{MARCO TEÓRICO}

\section{El ámbito jurídico de los Mecanismos Alternativos de Solución de Controver- sias}

En México, toda actividad profesional parte de un fundamento, de una formalidad, de una normatividad, de una regulación profesional. Siendo así, para el profesional de los mecanismos alternativos es imprescindible el conocimiento y reconocimiento del plano constitucional desde donde participe en esta esfera profesional, para con ello fortalecer la demanda de justicia social, lógicamente en un contexto diferente al que se concebía de la imperatividad de intervención judicial original como lo fue hasta la incorporación de esta vía, que si bien también atiende a un procedimiento, lo hace bajo principios que favorecen las condiciones de acordar de las partes no vistas en disputa como antaño. Esto es precisamente lo que se enmarca heurísticamente en nuestra Carta Magna dentro de su artículo 17, que en su párrafo cuarto dispone: "las leyes preverán mecanismos alternativos de solución de controversias". Lo anterior, también se asume en las diferentes entidades del país desde sus constituciones locales y legislaciones especializadas respectivas bajo la misma perspectiva que lo formaliza. En el caso del estado de Nuevo León, se ubica dentro de su artículo 16 donde se describe que toda persona bajo esta jurisdicción tiene el derecho a resolver sus diferencias mediante estos mecanismos en la forma y términos establecidos por las leyes concernientes, que, para los efectos, como anteriormente se manifestó existe tal ley especializada.
Siendo así, todo impulso en las legislaciones parte de las necesidades sociales donde se tiene como precedente de motivación en su contexto teórico y conceptual, lo que acontece con el fin de regular y normar la convivencia entre personas, y las desavenencias que surgen entre sí, de tal manera que se encuentre una plataforma de necesidad para atender las mismas en estos canales donde el deber conduce a una certeza jurídica por el fundamento normativo existente, tanto como por el contexto del deber hacer y quehacer en el mismo.

En este sentido, comprendiendo los mecanismos alternativos de acuerdo con la Ley de Mecanismos Alternativos para la Solución de Controversias del Estado de Nuevo León en su artículo $2^{\circ}$, fracción XX, donde los señala como procedimientos distintos a la justicia ordinaria que permiten prevenir, abordar y solucionar controversias de manera voluntaria y colaborativa, pudiendo ser en forma presencial o a distancia mediante el empleo de tecnologías de la información y la comunicación; de la misma manera en su fracción $V$ del mismo apartado señala la certificación especializada como la constancia otorgada por el Instituto de Mecanismos Alternativos para la Solución de Controversias del estado para acreditar que una persona física cuenta con los conocimientos, competencias y habilidades necesarias de tal forma que pueda desempeñarse como facilitador, mediante una formalidad esencial del procedimiento, pero que a la vez cuenta con los conocimientos jurídicos suficientes para que los convenios resultantes de los mecanismos alternativos que se celebren bajo su intervención se eleven a la categoría de cosa juzgada o 
sentencia ejecutoriada en su caso, bajo los términos de la presente Ley.

En el mismo orden, bajo lo preceptuado en el apartado XIV respecto del concepto de facilitador, del cual manifiesta es el responsable de atender el procedimiento señalado, reconociéndose como la persona física que cuenta con certificación o certificación especializada del Instituto para prestar los servicios de mecanismos alternativos respectivos, y que podrá ejercerlos, ya sea como árbitro, conciliador o mediador ya sea en el propio instituto, en los centros de mecanismos alternativos acreditados en los términos de esta Ley, o en forma independiente. Hasta aquí es preciso detenerse puesto que esta revisión legal debe robustecer la razón de que la persona física, se entiende profesional, de dichos mecanismos, se encuentre certificada bajo las competencias profesionales homologadas que posicionen el compromiso en este proceso que se avala desde la Carta Magna, como previamente se señaló, tanto como de las leyes que los regulan.

\section{La disciplina profesional, en un enfoque homologado por los mecanismos alterna- tivos}

Bajo la perspectiva citada de revisión doctrinaria, es importante partir del concepto genérico de disciplina, pero de aquella que conduce hacia el punto en común con la respectiva de los mecanismos alternativos. Dicho de otro modo, si bien tales mecanismos parten de un ordenamiento jurídico, esto no se encuentra limitado a los abogados, por lo que es de importancia recalcar la posibilidad de intervención por parte de la variedad de ciencias, disciplinas o áreas del conocimiento que se concentran con un mismo propósito y normas, puesto que los conflictos tienen una amplia variedad de aparición. Es decir, no se circunscriben únicamente a actos o hechos jurídicos que sólo los abogados pueden comprender, sino que se requiere en su caso de personas con conocimientos especializados de la rama donde aparezca el conflicto. Cabe aclarar el hecho de que tales áreas del conocimiento no las circunscribe a llevar a cabo operaciones periciales, sino que como los métodos alternos no exigen una limitación a la formalidad esencial del procedimiento para redimir el conflicto, esto hace que tales especialistas del conocimiento puedan encontrarse en una posición técnicamente más cercana a efecto de preparar las condiciones que lleven a la solución de la (s) controversia (s).

Siendo así, es de acentuar que el concepto de disciplina posee varias acepciones. Entre estas, se le conoce como el principio de arte, facultad o ciencia, o acatamiento debido al mandato, orden o norma legítimos (De Pina, 2013). Así también, como un conjunto de reglas de comportamiento para mantener el orden y la subordinación entre los miembros de un cuerpo o una colectividad, de acuerdo con el Diccionario Enciclopédico de Ciencias de la Educación (2000). Hasta ahora, se concluye que la disciplina se trata de orden, de atender normas y principios de acuerdo con determinada ciencia o disciplina del conocimiento sistematizado.

De acuerdo con lo anterior y enmarcando a una posible disciplina dentro del ámbito de los mecanismos alternativos, existen expertos de variadas disciplinas y campos de conocimiento, quienes pueden asumir el carácter de facilitador con un deber de conducir la co- 
municación, o conciliar en su caso, entre las partes en conflicto con el fin de llegar a un acuerdo, pero sobre todo que, en perspectiva, beneficie a las partes.

Por lo tanto, es de destacar un valor anexo en la actividad profesional conocido como "interdisciplina", cuya característica es que puede ser descrita de la siguiente manera:

conjunto de disciplinas conexas entre sí, con relaciones definidas, a fin de que sus actividades no se produzcan en forma aislada, dispersa y fraccionada, sino en un contexto de verticalidad de la ciencia o del conocimiento que está dado por cada especialidad particular, $y$ es considerada como una forma de ver, acercarse, conocer y tratar un problema (Tamayo, 2006) razón de que se interactúa con diversas disciplinas por el uso de conocimientos diversos integrándolos de manera contextualizada y sistemáticamente para la solución de un conflicto (Gorjón, 2017).

Es mediante este paradigma donde las disciplinas convergen en un mismo plano, como lo es la solución de problemas de seres humanos que piensan y actúan de manera independiente, pero controversial. Respecto a lo antes señalado, es aquí donde el facilitador o profesional competente, pero acreditado, deberá trabajar con dos partes opuestas, que en su calidad o juicio de valor personal no afecte el procedimiento y pueda con ello evidenciar una debilidad en su competencia profesional que afecte las demeritadas relaciones de las partes que se encuentran bajo su compromiso profesional, en virtud que tales partes acuden voluntariamente para que expertos, a quienes les confían su historia y posicionamiento ante ello de las acciones que los conducen a tener diferencias y que estas forman la causa natural de generador de conflictos, es que buscan a través del profesional de los mecanismos alternativos la mejor opción de solución del respectivo conflicto.

Es de aclarar doctrinariamente, y se coincide con esta afirmación, que

los mecanismos alternativos se sustentan en su capital intelectual, al no tratarse sólo de un valor económico, sino más bien, se trata de un ejercicio de gestión del conocimiento más allá de visualizar únicamente valores morales, legales o filosóficos, limitados por el actual normativismo; sino por un ejercicio intelectual, innovador y creativo que trasciende las fronteras de lo tangible que se centra en los intereses (Gorjón, 2017)

Por lo tanto, la suma de aportaciones intelectuales en los mecanismos alternativos, se distingue no sólo por la particularidad de cada disciplina como interviniente, que para el caso sería facilitador, sino que se encuentra en la variedad de formaciones profesionales, de disciplinas diversas, ya sea de las ciencias sociales o de las ciencias exactas, con perfiles profesionales cuyos valores profesionales son distintos, pero coincidentes en un común denominador, en una competencia profesional y personal, con el deber de poseer fortalezas profesionales y personales que faciliten la comunicación y lleven al entendimiento entre las partes. 
Es por lo antes descrito que la diversa profesión que tenga como metal la solución de controversias a través de mecanismos alternativos, claro, desde cada disciplina donde interviene, debe encontrarse homologada partiendo de una formalidad, es decir, de un reconocimiento judicial que regule dichos actos (De Pina, 2013). Para tal efecto, debe ser el propio órgano normativo de los mecanismos aludidos quien lo sustente en principios base, a través de certificaciones que comprueben la competencia de esta actividad, cuyo propósito y fin principal es el de intervenir, prevenir y tener de propósito un acuerdo de paz, el cual debe traducirse desde sus respectivas panorámicas, en un ámbito idóneo ante los conflictos, con expectativas de mantener un contexto de respeto a las relaciones interpersonales.

Para cumplir con el objetivo anterior es necesario considerar ocho principios destacados en el ordenamiento jurídico de los mecanismos alternativos: confidencialidad, equidad, flexibilidad, honestidad, independencia imparcialidad, neutralidad y voluntariedad. Tales preceptos como atributos profesionales, indistintamente de la disciplina donde se atienda a las partes en conflicto, deben poseer además cualidades de valor, como lo es el promover el respeto entre las partes, dirigirse con ecuanimidad, destacar las habilidades e inteligencia emocional del profesional de los mecanismos alternativos; que se les permita dirigir a las partes en la búsqueda de soluciones, que el conflicto se exponga en un contexto de paz y neutralidad, y sobre todo, que no empeore el ánimo de las partes; que en una posición idónea estimule la comunicación y localice desde una forma introspecti- va las limitaciones que perfilan su persona, su propia disciplina y formación. Todos estos deberes sólo se habrán de conformar si se homologan, o equiparan con el conocimiento de cada parte del procedimiento y fin de los mecanismos alternativos, lo cual debe ser proporcionado y establecido por personas que se hayan especializado específicamente en los métodos alternos de solución de controversias.

Bajo un enfoque de realidad, es de destacar que en la práctica de la disciplina señalada, como en cualquier otra disciplina o ciencia, existen intrusismos disciplinares, es decir, con carencias de ética y valores, que mezclan otras disciplinas, o bien, que adolecen de fundamentos científicos para la atención de conflictos, puesto que al no contar con el perfil o formación profesional dentro de un enfoque de relaciones humanas, o en su caso cometer un desacierto como el confundir el papel del profesional de los mecanismos alternativos con la aplicación de su ciencia y diciplina principal, como en el caso de quienes ejercen la actividad profesional del psicoanálisis, donde se distorsiona la actividad asumiendo su función como proceso terapéutico, o como el abogado atiende a las partes tal fuera un procedimiento litigioso, o del trabajador social que traslada la actividad de facilitador a un trabajo de campo, o del criminólogo en una investigación de delito, etc.

Con lo anterior, no se niega que estas disciplinas puedan conformar una actividad interdisciplinar, donde puedan dirigir un procedimiento en el ámbito de los mecanismos alternativos, pero lo que se enfatiza es que se mantengan en una sola perspectiva forma- 
da por su disciplina, ya que el procedimiento prevé la presencia de un facilitador en la atención de las partes en conflicto, salvo casos en particular que requieran intervención especializada, pero para el caso particular que puedan entrar en una co-mediación, siendo aceptable tal trabajo donde se sume la experiencia de múltiples disciplinas y que estas se agreguen a la causa de la solución de controversias. Por tanto, hasta aquí, se pueden observar dos las virtudes que implican la interdisciplina.

Así las cosas, entre la disciplina profesional y el rol del facilitador reconocido y homologado por el Instituto de Mecanismos Alternativos, debe delinearse con rigor el requisito profesional para acceder a protestar dicho papel, cuya actividad reiteradamente conlleva un alto compromiso y responsabilidad.

Por tal razón, es necesario considerar que, conforme a la participación de variadas disciplinas, en casos de preparación como facilitadores, todo esto debe encontrarse alineado a un propósito y bajo su respectiva norma, con un mismo fin, de tal manera que se acumule cada experiencia profesional al ámbito de los mecanismos alternativos, sin desatender desde luego su propio contexto disciplinar en una sola línea de procedimiento.

Por consiguiente, el enfoque de esta disciplina donde se vislumbra un plano homologado en su quehacer es un punto de partida que, si bien se puede percibir complicado, esto no cataloga una imposibilidad de atención. Una primera visión es que aun sin ser fácil trasladar un perfil profesional determinado de una disciplina específica al de una enfocada al campo de la resolución de conflictos, si se revisa el ámbito y contexto jurídico de los mecanismos alternativos, se puede observar que se encuentra conceptualizada cada parte interviniente sin hacer discriminación, es decir, está enmarcada cada acción de los principios que enmarcan el procedimiento, lo que implica que al acatar tales disposiciones de competencias y conocimientos profesionales en este marco referencial, se puede cumplir con describir los componentes de los mecanismos alternativos cumpliendo con la formalidad, pero también con el fondo del ser y hacer profesional.

\section{La competencia profesional del facili- tador}

Este término se refiere específicamente a la competencia que tiene a su cargo un facilitador en el campo de los mecanismos alternativos, la cual se identifica con la realización y modo de trabajo sobre una actividad determinada, integrando las habilidades y los conocimientos propios de su disciplina, así como de los valores profesionales, y aunque es de admitirse, las emociones personales, todo lo cual puede en un momento dado regular el desempeño del profesional, cuyo propósito en común debe ser la búsqueda de soluciones a las dificultades profesionales. Es por ello, entre otras cosas la importancia de la homologación propuesta.

Ahora bien, pasando a las competencias profesionales, estas se comprenden como la suficiencia en capacidades, habilidades o destrezas que una persona adquiere a partir de un aprendizaje desde una determinada disciplina científica con el fin de aplicar en una actividad específica previamente con- 
traída por medio de un compromiso. En este sentido, la preparación parte desde la incorporación de competencias básicas acreditadas en un currículo, lo cual exige el deber de plantearse sobre cuáles son los aprendizajes fundamentales que se debe adquirir para la resolución de problemas a los que se enfrenta un profesional (Tunning, 2003).

Por su parte, Camellas (2000), destaca que la competencia de una persona, desde un campo determinado, se atribuye porque pone en juego diferentes capacidades necesarias con el fin de ofrecer una respuesta global a la situación que se le plantea, y en el mismo sentido señala que la posesión de competencias determina la globalidad del comportamiento del profesional ante los requerimientos, no dando respuestas rutinarias ni automáticas.

Otra aportación en el mismo sentido es la de Hortal (2002), quien señala que el profesionista, al adquirir los conocimientos y las habilidades que lo distinguen como tal, también adquiere el compromiso y la responsabilidad de prestar bien y de manera eficiente el servicio o bien que le compete y por el cual la sociedad lo acepta y reconoce como profesionista. Es así que a partir de que un individuo ejerce profesionalmente su disciplina formativa, se puede considerar que se encuentra socialmente inmerso en un medio laboral, o bien en una actividad determinada como en el caso del profesional en los mecanismos alternativos, siendo precisamente en este medio donde el término de competencia surge en su deber y quehacer, conociéndose como un atributo otorgado al individuo por la sociedad basado en su campo de conocimiento, tras considerar todas las interacciones del individuo, y toda vez que se cumpla con las expectativas de la sociedad y del cliente, generando una funcionalidad con conservación de su identidad laboral en el tiempo (Ruiz, 2008). Es precisamente en este esquema donde se apertura otro elemento de la homologación, porque se relaciona con el compromiso que todo profesional le debe a la sociedad como compensación del reconocimiento de un estatus social de intelectual.

Otro aspecto a ser notar es la importancia de aludir al término de la concepción de la profesión donde Hortal (2002) concibe a las profesiones. Para él estas son actividades ocupacionales en las que de forma institucionalizada se presta un servicio específico a la sociedad por parte de un conjunto de personas identificadas como profesionales, quienes se dedican a ella de forma estable obteniendo como resultado un medio de vida, y donde se coincide en la afirmación de que es justo en el campo del ejercicio de la profesión puesto que se demandan las exigencias de un debido cumplimiento, observándose las prácticas de la profesión, el trabajo en equipo, la necesidad de conocimientos y sobre todo las limitaciones o problemas que se hayan padecido. Sin embargo, no se percatan en ciertas ocasiones del proceso de formación (Benavides, 2009), razón de que la disciplina profesional debe vincularse a la exigencia de determinada actividad, profesionalizando la misma y que ésta fundamente la formación del profesional y a su vez brinde la certeza en la calidad del servicio que se espera. Una forma de hacer que se percaten de su concepción de formación del profesional es precisamente donde se les inspi- 
re a que la solución de los problemas de su ciencia no sólo se presenta de manera aislada, sino que, conforme a la doctrina de la filosofía de la paz, es como puede percibir la otra esfera paralela a su actuar, y que al darle importancia lo vuelve más holístico en la solución de los problemas.

En el mismo contexto, sobre estas exigencias, la aportación de Bunk (1994) es significativa, ya que para este autor la transmisión de las competencias (mediante acciones de formación), se basa en la acción, donde el desarrollo de la competencia integrada (competencia de acción), requiere de una formación o de un desarrollo (según se trate), pero dirigida a la acción; es decir, puede y debe relacionarse con las situaciones de trabajo con el fin de que la competencia cobre su sentido genuino y global. De este modo, en los procesos de formación basados en competencias, los procesos de aprendizaje que se favorecen deben orientarse hacia la acción del participante, tomando como referente el marco organizativo en el que la situación de trabajo es situación de aprendizaje como círculo virtuoso donde nunca se deja de aprender, y es precisamente que la formación sobre los mecanismos alternativos de solución de controversias estimulan además de la solución del problema, a la cavilación del sentido de satisfacción obtenida por haber participado en la construcción de la solución respectiva.

No obstante, es menester regresar por lo pronto a la competencia donde se formen expectativas externas, debiendo por ello cumplir con la respectiva formación de profesional que lo califique como competente, sin perder la orientación a los requerimientos y normati- vas de su propio quehacer, pero de lograrse esto, se puede preparar al profesional a trascender en su conocimiento de logros.

Lo anterior conduce al ámbito de la certificación profesional. En torno a este concepto Bertrand (2000), la define como el proceso a través del cual se aseguran las competencias y las habilidades de un individuo en relación con una norma formalizada. Se trata por ello de la certificación de unas cualificaciones individuales, de un nivel de conocimientos, de unas habilidades $y$, probablemente, de unas capacidades de aprendizaje. Las certificaciones de dichos profesionales avalan capacidades de intervención y atención ante problemas que aquejan a las partes en conflicto. Es así como:

la especialización en cada dominio conlleva nuevos progresos; ésta es necesaria cuando una disciplina evoluciona, ya que permite el análisis profundo de un objeto de estudio determinado, posibilitando una mejor comprensión de los fenómenos examinados. A medida que una mirada teórica se expande, sus practicantes se especializan cada vez más (Dogan \& Pahre, 1993).

Por lo tanto, es que cuando se trata de enunciar habilidades, conocimientos específicos e inmiscuidos los valores profesionales, se puede considerar el haberse consolidado una competencia, no sin antes señalar que cada desempeño de determinada disciplina específicamente en el campo de los mecanismos alternativos, se pueden observar variaciones en su quehacer, en virtud que dependen de su experiencia y de su disciplina principal, 
donde es evidente que iguales competencias podrían tener desempeños y resultados diferentes, y como explicación a ello lo encontramos en su perfil y las variables del entorno.

Es razón de esto, el desarrollar la capacidad de un profesional a través del aprendizaje continuo, es dar una respuesta a la necesidad de la sociedad en la búsqueda y solución de sus controversias, coincidiendo con lo expresado por la Organización de las Naciones Unidas para la Ciencia y la Cultura (UNES$\mathrm{CO}$ ) en cuanto a que los pilares de la educación y del aprender comprenden: "Aprender a conocer, aprender a hacer, aprender a vivir juntos, aprender a ser" (Forgas, 2002).

Siendo así, el profesional de los mecanismos alternativos su deber es hacer, pero también debe aprobar la competencia del profesional, donde avale y verifique el campo teórico y práctico de poseer cualidades en atención al campo de la solución de controversias, y que estas conduzcan hacia convenios aceptados y homologados por la respectiva autoridad judicial, acciones que formalizan los acuerdos finales con el fin de garantizar jurídicamente el cumplimiento por las partes involucradas. Por lo antes señalado, cabe mencionar que la Ley de Mecanismos Alternativos para la Solución de Controversias del Estado de Nuevo León, la cual describe el término de certificación, dirigida a facilitadores, lo señala en su fracción IV, así como de la especialización, fracción $V$ respectivamente. Donde se establece que la "certificación" es la constancia otorgada por el Instituto para acreditar que una persona física, cuenta con los conocimientos, competencias y habilidades necesarias para desempeñarse como facilitador de conformidad con el presente ordenamiento; $y$ por certificación especializada se identifica a la constancia otorgada por el Instituto para acreditar que una persona física cuenta con los conocimientos, competencias y habilidades necesarias para desempeñarse como facilitador, de conformidad con el presente ordenamiento, y que además cuenta con los conocimientos en el campo del Derecho suficientes para que los convenios producto de los mecanismos alternativos que se celebren bajo su intervención se eleven a la categoría de cosa juzgada o sentencia ejecutoriada, en los términos de la presente Ley; destacando en ambos apartados que se documenta la acreditación, salvo en la segunda que dicta el carácter de certificación especializada donde se le aporta tal atribución, de que todo convenio que se realice se haga con la certeza jurídica de cumplimiento por darse la categoría de cosa juzgada o sentencia.

De acuerdo a lo expuesto, en dicho apartado jurídico se entiende que para el logro de la obtención de los certificados se debe acreditar la competencia delineada desde su reglamento, a través de la conformación de un comité evaluador, donde se agrega a esto el requisito de que se efectúe bajo los procedimientos jurídicos regulados, que unifiquen el actuar y desempeño del facilitador profesional en la atención de situaciones en conflicto, desde sus distintas materias a resolver bajo su encomienda. Pero ello queda bajo observancia de autoridades designadas por el Instituto de Mecanismos Alternativos para la Solución de Controversias pertenecientes al del Poder Judicial del Estado de Nuevo León (Fracción XVII). 
Sobre el trámite anterior, se estableció mediante una reforma del año 2017 que se deberá contar con diploma o constancia expedida por el Instituto de Mecanismos Alternativos para la Solución de Controversias del Poder Judicial del Estado de Nuevo León, de alguna institución educativa o algún centro de mecanismos alternativos que cuenten previamente con la constancia de aprobación expedida por el primero de estos, de que sus programas de capacitación y formación, cumplen lo previsto en el artículo 35 de la Ley ya referida. Lo precedente, a fin de acreditar las 72 horas de capacitación teórico-práctica en mecanismos alternativos a que hace alusión el artículo 52 del reglamento de la ley antes citada. Asimismo, y agregando a este requisito se le solicita al aspirante: 1.- aprobar en primer lugar, una evaluación teórica con un mínimo de 80 puntos, en una escala de 100; y 2.- aprobar una evaluación práctica también con un mínimo de 80 puntos, en una escala de 100 .

Por lo antes descrito, se observa que el proceso de evaluación se encuentra equiparado a un procedimiento a cumplir, indistintamente la disciplina profesional que demuestre, ya que no se incluye el requisito de contar con una profesión o disciplina específica, sólo destacan que los facilitadores, de acuerdo al artículo 34 del mismo ordenamiento local, estos serán personas físicas y podrán ejercer esta función en la modalidad respectiva, dentro del Instituto de Mecanismos Alternativos, en los Centros de mecanismos alternativos acreditados, o desarrollar su actividad en forma independiente, debiendo acreditar que cuentan con estudios en mecanismos alternativos aprobados por el Instituto, también cumplir con los demás requisitos determinados en la Ley y su Reglamento.

Por otro parte, la Ley Nacional en Mecanismos Alternativos para la Solución de Controversias en Materia Penal (art. $3^{\circ}$ ), señala como requisito que el facilitador para ingresar a dicho órgano, se debe encontrar debidamente capacitado y certificado, cumpliendo con 180 horas de capacitación teórico-práctica en los MASC y renovando su certificación cada tres años, con 100 horas de capacitación, según lo advierte el artículo 50 de la ley nacional aludida. Así mismo, se delimita a la aceptación, no de persona física como lo dicta la normativa local, sino que especifica en su artículo 48 fracción I, que para ser facilitador deberán: poseer grado de Licenciatura afín a las labores que deberán desarrollar, con cédula profesional con registro federal; y de la misma forma se solicita la acreditación de la certificación que establece dicho ordenamiento.

Es de destacar que se cuenta con una clara normatividad, con bases que blindan la legalidad del facilitador, que se conforma bajo la premisa donde se configure la competencia profesional y que esta responda a la necesidad del contexto que solicita la sociedad en estas tareas donde deberán actuar bajo principios profesionales.

En razón de lo anteriormente expuesto, significa todo un reto la persistencia en la homologación de la profesionalización de los mecanismos alternativos y se coincide con la afirmación de que se trata de desentrañar la profesionalidad para fragmentarla en diferentes competencias que, al mismo tiempo dan respuesta a un concepto completo y glo- 
bal de la cualificación, para así permitir sortear los importantes problemas metodológicos y éticos asociados a la valoración de esos escurridizos elementos singulares (Baigorri, Martínez y Monterrubio, 2006: 41).

\section{CONCLUSIONES}

En el presente artículo se ha destacado los elementos necesarios en relación al constructo de la competencia profesional homologada en la actividad de los mecanismos alternativos, argumentos vinculados al marco conceptual de la profesión vista desde su quehacer, deber ser y hacer, basado en el compromiso de una capacitación que genere competencias profesionales que permitan resultados positivos en la resolución de conflictos, ante esto, mostrando que deben fundarse en principios generales que conduzcan a la integración de elementos que en base a la conciencia personal sobre el compromiso, se logre la versatilidad de las profesiones, dirigida a resolución de conflictos, considerando en ello las necesidades y el vertiginoso cambio del contexto normativo y social, lo cual impulsa la profesionalización.

En este mismo apartado, se ha planteado de acuerdo al marco teórico que lo fundamenta que las profesiones se distinguen por su conocimiento, por una cultura y perfil especial distintivo, que posee variaciones en su coherencia, donde depende del contexto social y la demanda de necesidades de los servicios profesionales, las cuales tendrán que asumir compromisos de actualización que respondan a una sociedad en movimiento, con transformaciones que propician la incorporación de la suma de otras disciplinas y que estas sean homologadas bajo acreditaciones de competencias específicas y de una actualización continua.

Como propuesta, es de señalar que a partir de la profesionalización, disciplina y competencia del facilitador en los mecanicismos alternativos, es el mantener la constancia del proceso y evolución intelectual en el plano de la intervención en la solución de controversias, que implique bajo un mismo orden, una sistematización metodológica del conocimiento, es decir, una formación única del profesional cuya exigencia sea el contar con un grado universitario, que le genere competencias y licencia para que de manera automática, el facilitador aporte bajo sus conocimientos curriculares, no sólo de cursos teóricos y prácticos, sino de un diseño de estrategias y tácticas dependiendo de la situación donde intervenga; todo lo cual es inherente a una formación profesional de nivel profesional superior. Por ende, debería ser requisito del aspirante a facilitador el prepararse continuamente, dependiendo del ámbito de aplicación en los métodos alternos.

Una segunda propuesta es la homologación de requisitos en la figura del facilitador desde la Ley Nacional y la legislación local, ya que en la primera solicita sea un profesional con grado de Licenciatura, certeza de una formación intelectual y de conocimientos que pueden acercarlo a la actividad en particular, caso opuesto en la legislatura local que señala a los facilitadores sin el requisito de una academia profesional, donde sólo lo delimita a personas físicas. 
Siendo así, la homologación como reconocimiento legal sobre la regularidad de un acto jurídico, mismo que representa el procedimiento inmerso en los mecanismos alternativos, clarifica el posicionamiento del profesional llamado facilitador, adjudicándole una representación sólida, indistintamente la disciplina que le da origen al impulso de hacer de esta actividad su desarrollo.

\section{TRABAJOS CITADOS}

Baigorri López, J., Martínez Cía, P., \& Monterrubio Ariznabarreta, E. (2006). El reconocimiento oficial del saber profesional obtenido por la experiencia. Revista Europea de Formación Profesional CEDEFOP. (37) pp. 36-54.

Benavides Martínez, B. (2009). Contexto social de la profesión. México, D.F.: Patria.

Bertrand, O. (2000). Evaluación y certificación de competencias y cualificaciones profesionales. Madrid: OEI.

Bunk, G. P. (1994), La transmisión de competencias en la formación y perfeccionamiento profesionales de la RFA. Revista Europea de Formación Profesional. (1) Pp. 8-14. Recuperado en: https://dialnet.unirioja.es/ejemplar/13171

Comellas, M. J. (26-28 junio 2000) Las competencias profesionales de la formación en Psicopedagogía: objetivo fundamental de la docencia universitaria. Comunicación del I Congreso Internacional: Docencia Universitaria e Innovación. Barcelona.

Constitución Política de los Estados Unidos Mexicanos.

Constitución Política del Estado de Nuevo León.

De Pina Vara, R., \& De Pina García, J.P. (2013). Diccionario de Derecho, México, D.F.: Porrúa.

Dogan, M., \& Pahre, R. (1993). Las nuevas ciencias sociales: la marginalidad creadora. México. D.F.: Grijalbo.

González J., \& Wagenaar, R. (Eds.) (2003). Tunning, Educational Structures in Europe. Final Report. Pilot Proyect. Phase One. Bilbao. España: University of Deusto, University of Groningen. Recuperado en: http:// http://tuningacademy.
org/wp-content/uploads/2014/02/TuningEUI_Final-Report_SP.pdf

Gorjón Gómez, F. J. (2017). Mediación, su valor intangible y efectos operativos. Una Visión Integradora de los Métodos Alternos de Solución de Conflictos. Ciudad de México: Tirant Lo Blanch.

Hortal Alonso, A. (2002). Ética General de las Profesiones. Bilbao, España: Desclée.

Ley Nacional de Mecanismos Alternativos de Solución de Controversias en Materia Penal.

Ley de Mecanismos Alternativos para la Solución de Controversias del Estado de Nuevo León.

Picardo Joao, O., Escobar Baño, J. C. \& Pacheco Cardoza R. B. (2004). Diccionario Enciclopédico de Ciencias de la Educación. Barcelona: CEAC. San Salvador. El Salvador: Centro de Investigación Educativa, Colegio García Flamenco.

Ruiz Barría, G. (2008). Reflexiones y definiciones desde la teoría biológica del conocimiento: aprendizaje y competencia en la universidad actual. Revista Estudios Pedagógicos. 34 (1) 199-214. Recuperado en: http://revistas.uach.cl/ pdf/estped/v34n1/art12.pdf

Tamayo y Tamayo, M. (2006). La Interdisciplinariedad. Cali. Colombia: ICESI. Recuperado en: https://repository.icesi. edu.co/biblioteca_digital/bitstream/10906/5342/1/interdisciplinariedad.pdf

\section{Raquel Rodríguez}

Doctora en Derecho, con Orientación en Derecho Procesal, Maestría en Métodos Alternos de Solución de Controversias, Maestría en Criminología Lic. en Derecho y Ciencias Sociales por la Universidad Autónoma de Nuevo León, México; Diplomados en: Psicología Positiva y Psicología Jurídica, Derecho del trabajo y Violencia de Género. Miembro del Colegio de Mediadores de Nuevo León. Líneas de investigación: Mecanismos de Solución de Controversias, Derecho del trabajo y Violencia de Género. Profesora de tiempo completo de la Universidad Autónoma de Nuevo León, México. 